\title{
Azithromycin Treatment Failure Among Primary and Secondary Syphilis Patients in Shanghai
}

\author{
Pingyu Zhou, MD, PhD*, Kang Li, MS*, Haikong Lu, MD*, Yihong Qian, MD*, Xin Gu, MD*, \\ Weiming Gong, MD*, Joseph D. Tucker, $\mathrm{MD}^{\dagger}$, and Myron S. Cohen, MD ${ }^{\ddagger}$ \\ * Department of STD Institute, Shanghai Skin Disease \& STD Hospital, Shanghai, China \\ † Guangdong Provincial Dermatology \& Venereology Center, Guangzhou, China \\ ₹ School of Medicine, University of North Carolina, Chapel Hill, NC
}

\begin{abstract}
Background-Azithromycin has been used to treat primary and secondary syphilis and as prophylaxis for sexual partners. We evaluated syphilis treatment failure in patients who received azithromycin therapy.
\end{abstract}

Methods-Patients who did not respond to azithromycin therapy were referred to Shanghai Skin Disease and sexually transmitted disease hospital. Treatment failure was defined as follows: (1) persistent ulcers or cutaneous or mucosal lesions 1 month after therapy; or (2) detection of spirochetes in dark-field microscopy examination of a lesion at least 1 week after treatment; or (3) failure of rapid plasma reagin titers to decrease 4-fold at 3 months after treatment.

Results-A total of 132 patients with primary and secondary syphilis who failed azithromycin therapy were referred to our hospital between January 2001 and October 2008. Of 132 patients, 42 $(31.8 \%)$ had primary syphilis and $90(68.2 \%)$ had secondary syphilis. Twenty-six patients with primary syphilis developed multiple lesions or secondary syphilis, or persistent ulcers despite using azithromycin. The skin or mucosal lesions did not resolve in 37 patients with secondary syphilis after azithromycin treatment. Ten patients had a positive dark-field examination for Treponema pallidum (T. pallidum) after treatment. The serum rapid plasma reagin titers studied in all cases had failed to decrease 4-fold at 3 months after therapy. The doses of azithromycin used for treatment ranged from 4 to $30 \mathrm{~g}$.

Conclusions-The failure of azithromycin to cure a substantial number of patients with primary and secondary syphilis in Shanghai suggests that azithromycin has limited therapeutic value in this setting.

Syphilis is increasing in China. In 2008, 278,215 cases of syphilis were reported, including 138,667 cases of primary and secondary syphilis, with an incidence of 21.06 cases per 100,000 population. The situation of syphilis is of particular concern in Shanghai, where, in 2008 , the incidence of syphilis reached its highest level of 76.78 cases per 100,000 population, the second in the nation. 14,266 cases were reported and 9073 cases were diagnosed as primary and secondary syphilis. ${ }^{1}$

Prompt detection of infection and effective treatment are key elements to the control of syphilis. Penicillin has remained the recommended treatment for syphilis since its discovery

Copyright (C) 2010 American Sexually Transmitted Diseases Association All rights reserved.

Correspondence: Pingyu Zhou, MD, PhD, Department of STD Institute, Shanghai Skin Disease \& STD Hospital, 196 Wuyi Rd,

Shanghai 200050, China. zpyls@yahoo.com.

P.Z. and M.S.C. contributed equally to this work. 
more than 60 years ago. But penicillin requires special storage conditions and intramuscular or intravenous delivery, and can cause severe allergic reactions. Accordingly, a safe and simple alternative therapy has been sought, especially in resources constrained countries. Azithromycin is an azalide (macrolide) antimicrobial agent with a long half-life (68 hours), good tissue penetration, and excellent activity against Gram-negative organisms. ${ }^{2-3}$ It has been successfully used as a single-dose, oral treatment for early syphilis. ${ }^{4-9}$ However, a Treponema pallidum ( $T$. pallidum) genotype consistent with macrolide resistance has been described in some patients with treatment failure, especially in patients with concomitant HIV infection. ${ }^{10-16}$ In this report, we describe a large number of patients with syphilis who failed azithromycin therapy.

\section{MATERIALS AND METHODS}

\section{Patients}

Between January 2001 and October 2008, sexually transmitted infection (STI) patients with primary or secondary syphilis were referred to our hospital and evaluated at our public STI clinic. These patients were originally treated with azithromycin because of allergy to penicillin or the shortage of penicillin, and they were believed to be potential azithromycin treatment failures. Patients with persistent high risk unprotected sex following syphilis treatment were excluded to reduce the possibility of reinfection. All the patients were evaluated by a physician and treated according to national Chinese STI treatment guidelines. ${ }^{17}$ Charts were reviewed to acquire demographic, clinical, and behavioral information. Clinical laboratory tests were also conducted, including initial dark-field microscopy, HIV, rapid plasma reagin (RPR) and T. pallidum hemagglutination (TPHA) test or fluorescent treponemal antibody absorption (FTA-ABS) test before and after azithromycin administration.

\section{Evaluation Criteria for Treatment Failure}

Azithromycin treatment failure after using minimum $4 \mathrm{~g}$ (maximum $2 \mathrm{~g}$ /day) of azithromycin was defined according to at least one of the following criteria: (1) persistent ulcers or cutaneous or mucosal lesions 1 month after treatment; (2) detection of spirochetes in a dark-field microscopy examination of a lesion at least 1 weak after treatment; (3) failure to decrease 4-fold for RPR titers within 3 months after treatment.

\section{Gene Mutation at 23S rRNA Gene of T. pallidum}

T. pallidum strains were screened for macrolide-resistance mutation by use of published methods. ${ }^{12}$ Briefly, swab samples were collected from primary lesions in 4 patients who had azithromycin treatment failure. DNA was extracted from swab samples using QIAmp DNA Mini Kit (QIAGEN, CA) following the manufacturer's instructions under stringent polymerase chain reaction (PCR)-clean conditions. Mutant isolates were identified using restriction enzyme analysis of PCR amplifications. A nested PCR and sequencing procedure were performed to confirm the gene mutation in T. pallidum DNA.

\section{RESULTS}

A total of 132 patients with primary or secondary syphilis were identified as azithromycin failure (Table 1). All patients had an appropriate response to ceftriaxone, procaine penicillin, and doxycycline therapy (Table 2). Most patients were male with an average age of 32 years (Table 3). Among 85 men with syphilis, 11 were MSM (men who have sex with men) and 4 were bisexual. Of the patients, $42(31.8 \%)$ had primary syphilis and $90(68.2 \%)$ had secondary syphilis. None of the 132 patients had a previous history of syphilis. All patients were tested for HIV with negative results. Forty-two patients with primary syphilis failed 
azithromycin therapy. Ten patients had a positive dark-field examination for T. pallidum after treatment. 37 had reactive TPHA or FTA-ABS tests and initial RPR titers ranged from 1:1 to 1:32. Among the 5 patients with negative RPR tests, 3 patients had a reactive TPHA or FTA-ABS tests and 2 showed negative results. Despite azithromycin therapy, persistent clinical manifestations were apparent in $26(61.9 \%)$ cases, including evolution from one to multiple ulcers (Fig. 1), other manifestations of secondary syphilis, and nonhealing ulcer (Table 1). A2058G mutations were found at $23 S$ rRNA gene of $T$. pallidum in all 4 cases (Fig. 2). The initially negative RPR in 5 cases turned positive, and TPHA tests of 2 patients who were nonreactive turned reactive. The remaining 16 patients had primary lesion resolution, but RPR titers at the follow-up visit remained within \pm 1 dilution of the initial RPR titer or increased $\geq 2$ dilutions 3 months after treatment (Table 1). The serum RPR titers in 42 primary syphilis cases ranged from 1:4 to 1:64 at their first follow-up visit after azithromycin treatment.

All 90 patients with secondary syphilis had reactive TPHA or FTA-ABS tests and the initial RPR titers ranging from 1:8 to 1:256. In 37 patients, skin lesions did not disappear 4 weeks after azithromycin therapy. Among 53 cases whose skin lesions disappeared, the serum RPR titers in 12 cases increased $\geq 2$ dilutions within 3 months and the RPR titers in other 41 cases remained within \pm 1 dilution of the initial RPR titer (Table 1).

All patients subsequently responded to treatment of procaine penicillin (37 cases), ceftriaxone ( 92 cases), or doxycycline ( 3 cases) both clinically and serologically. The serological and clinical responses are presented in Table 2. A total of 12 and 9 cases were lost to follow-up at 1 and 3 months, respectively.

\section{DISCUSSION}

Penicillin is the only antibiotic currently recommended for primary and secondary syphilis in non-penicillin allergic patients, but the storage, intramuscular administration, and the lifethreatening allergic potential of this drug greatly complicates the therapy. The antimicrobial spectrum and long half life of the azithromycin suggest its usage to treat syphilis. Several small trials demonstrated successful use of single dose azithromycin for primary and secondary and early latent syphilis. ${ }^{5-8}$ Two large clinical trials in Africa also demonstrated the efficacy of azithromycin treatment of syphilis. ${ }^{4,9}$

However, azithromycin treatment failures in syphilis were noted in San Francisco in 2002 among HIV infected men who had sex with men. ${ }^{10}$ A genotypic mutation (A2058G) that confers resistance to macrolides and azalides in T. pallidum has been described in isolates from San Francisco, Seattle, Baltimore, and Dublin (Ireland). ${ }^{11}$ An extremely high prevalence of the mutation (88.2\%) was found in Dublin, Ireland, and a moderately high prevalence in US cities (10.5\%-21.8\%). ${ }^{11}$ In San Francisco during 2004, azithromycinresistant $T$. pallidum strains represented $56.1 \%$ of isolates from all available patients treated with azithromycin for syphilis, compared to only 4.0\% during 2000-2002 and 40.6\% during 2003. ${ }^{12}$ A recent study in Shanghai found that all isolates obtained from 38 subjects included a A2058G mutation. ${ }^{13}$ The same scenario happened to all of the 4 patients in the current report whose specimens were analyzed for the mutation.

The majority of reported azithromycin failures in developed countries have been among MSM and those with HIV. ${ }^{10,12}$ However, in our report, $82.4 \%$ of the patients reported only heterosexual behaviors, and all subjects were HIV negative. Only $19.7 \%$ of this group of patients reported receiving an antibiotic in 6 months before therapy with azithromycin.

Nevertheless, azithromycin treatment failures and T. pallidum resistance have not been reported in Africa. Most published data on azithromycin therapy for syphilis in developing 
countries have shown generally good results, including those from large trials, although molecular studies have not been performed in these trials. ${ }^{4,9}$ However, some other reports and randomized clinical trials in China showed the failure of azithromycin in treating early syphilis or the various efficacy, from $55 \%$ to $92 \%$ at the same period of this study. ${ }^{14,18-21}$ These trials also showed the trend of rate increase of azithromycin treatment failure over time. Therefore, it is urgently needed to fully understand the current treatment situation in syphilitic patients with azithromycin and to monitor the macrolide resistance and gene mutation in T. pallidum in China.

This study has several limitations. First, the data lack of information about the percentage of patients treated with azithromycin during the study period in Shanghai or the whole nation, although we roughly estimated that the rate was about $0.1 \%$ to $5.0 \%$ in Shanghai. Second, the results provide no indication about the percentage of patients successfully treated with azithromycin. Third, compliance with azithromycin therapy was based on patient history, which might not be accurate. Fourth, the molecular study for treatment failure was only conducted in 4 patients.

The data of azithromycin treatment for syphilis and macrolide-resistant T. pallidum are very limited in China. We conducted a project to assess T. pallidum resistance in Shanghai, China, during 2007 to 2009, and found that, among 132 samples with T. pallidum, $126 \mathrm{had}$ gene mutations with a rate around $95.5 \%$ (unpublished data). It is widely believed that an increase of $5 \%$ in the prevalence of antibiotic resistance is an indicator to discontinue the particular antibiotics. Therefore, azithromycin might not be an option in treating syphilis in Shanghai, and the patients who have received azithromycin treatment must be closely followed up with clinical and serological evaluation for treatment efficacy.

In conclusion, azithromycin resistance in T. pallidum appears to be highly prevalent in Shanghai and is responsible for the high rate of treatment failure. Although more studies are required to fully understand the relationship among the treatment failure, macrolide resistance, and gene mutation in T. pallidum, we strongly suggest azithromycin should not be used to treat syphilis in the Shanghai area. Furthermore, until additional data are collected and further research is done, azithromycin should be used with caution to treat syphilis elsewhere in China and even East Asia.

\section{Acknowledgments}

The authors thank Dr. Sheila A. Lukehart, professor of University of Washington for kindly providing the 2 characterized strains of T. pallidum (Nichols and Street 14) and her technical support. The authors are also grateful to Mr. Shun Lv for his helpful comments and managing the tables and figures in this article.

\section{References}

1. Chinese Center for Disease Prevention and Control; Center for STI and AIDS Prevention and Control. 2008 report on syphilis and gonorrhea epidemic analysis in China. Bull STI Prev Control. 2009; 232:1-18.

2. Girard AE, Girard D, English AR, et al. Pharmacokinetic and in vivo studies with azithromycin (CP-62,993), a new macrolide with an extended half-life and excellent tissue distribution. Antimicrob Agents Chemother. 1987; 31:1948-1954. [PubMed: 2830841]

3. Foulds G, Shepard RM, Johnson RB. The pharmacokinetics of azithromycin in human serum and tissues. J Antimicrob Chemother. 1990; 25(suppl A):73-82. [PubMed: 2154441]

4. Kiddugavu MG, Kiwanuka N, Wawer MJ, et al. Effectiveness of syphilis treatment using azithromycin and/or benzathine penicillin in Rakai, Uganda. Sex Transm Dis. 2005; 32:1-6. [PubMed: 15614114] 
5. Verdon MS, Handsfield HH, Johnson RB. Pilot study of azithromycin for treatment of primary and secondary syphilis. Clin Infect Dis. 1994; 19:486-488. [PubMed: 7811868]

6. Campos-Outcalt D, Hurwitz S, Mickey T. Use of directly observed azithromycin treatment for syphilis in a homeless woman. Sex Transm Dis. 2002; 29:372. [PubMed: 12035029]

7. Hook EW III, Stephens J, Ennis DM. Azithromycin compared with penicillin G benzathine for treatment of incubating syphilis. Ann Intern Med. 1999; 131:434-437. [PubMed: 10498560]

8. Hook EW III, Martin DH, Stephens J, et al. A randomized, comparative pilot study of azithromycin versus benzathine penicillin G for treatment of early syphilis. Sex Transm Dis. 2002; 29:486-490. [PubMed: 12172535]

9. Riedner G, Rusizoka M, Todd J, et al. Single-dose azithromycin versus penicillin G benzathine for the treatment of early syphilis. N Engl J Med. 2005; 353:1236-1244. [PubMed: 16177249]

10. Centers for Disease Control and Prevention. Azithromycin treatment failures in syphilis infectionsSan Francisco, California, 2002-2003. Morb Mortal Wkly Rep. 2004; 53:197-198.

11. Lukehart SA, Godornes C, Molini BJ, et al. Macrolide resistance in Treponema pallidum in the United States and Ireland. N Engl J Med. 2004; 351:154-158. [PubMed: 15247355]

12. Mitchell SJ, Engelman J, Kent CK, et al. Azithromycin-resistant syphilis infection: San Francisco, California, 2000-2004. Clin Infect Dis. 2006; 42:337-345. [PubMed: 16392078]

13. Martin IE, Gu W, Yang Y, et al. Macrolide resistance and molecular types of Treponema pallidum causing primary syphilis in Shanghai, China. Clin Infect Dis. 2009; 49:515-521. [PubMed: 19583516]

14. Zhou P, Qian Y, Xu J, et al. Occurrence of congenital syphilis after maternal treatment with azithromycin during pregnancy. Sex Transm Dis. 2007; 34:472-474. [PubMed: 17589329]

15. Marra CM, Colina AP, Godornes C, et al. Antibiotic selection may contribute to increases in macrolide-resistant Treponema pallidum. J Infect Dis. 2006; 194:1771-1773. [PubMed: 17109351]

16. Morshed MG, Jones HD. Treponema pallidum macrolide resistance in BC. CMAJ. 2006; 174:349. [PubMed: 16446481]

17. Chinese Centers for Disease Control and Prevention. Sexually Transmitted Diseases Treatment Guidelines. China, Beijing: Chinese Centers for Disease Control and Prevention; 2007. p. 1-16.

18. Xi H. Penicillin G compared with azithromycin or ceftriaxone for treatment of early syphilis [in Chinese]. Guangxi Yi Xue. 2003; 25:1867-1871.

19. Yang LG, Zhang XB, Zhong S. Efficacy of azithromycin in treatment of early syphilis (report of 14 cases) [in Chinese]. Zhongguo Re Dai Yi Xue. 2004; 4:771-772.

20. Qiao G, Li Z, Liu LH. Study on the clinical therapeutic effective of three methods in patients with early syphilis [in Chinese]. Zhongguo Xing Ke Xue. 2005; 4:11-12.

21. Wu RX, Bi YL, Zhang YH. Analysis of the serum changes in the patients suffering from syphilis in the early stage after taking azithromycin [in Chinese]. Guangdong Yao Xue Yuan Xue Bao. 2006; 22:98-101. 


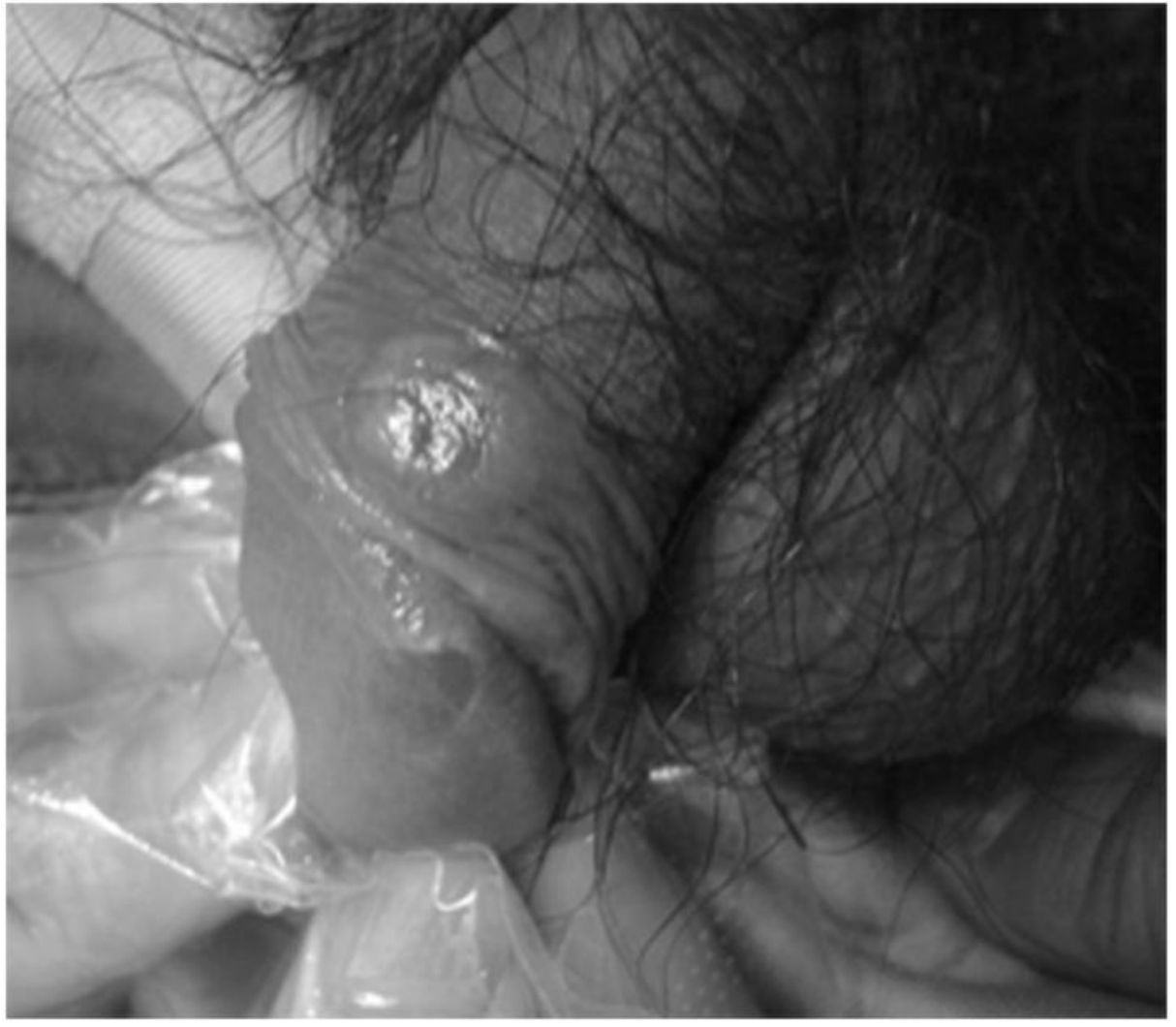

Figure 1.

Single chancre evolved to multiple lesions after azithromycin treatment. 


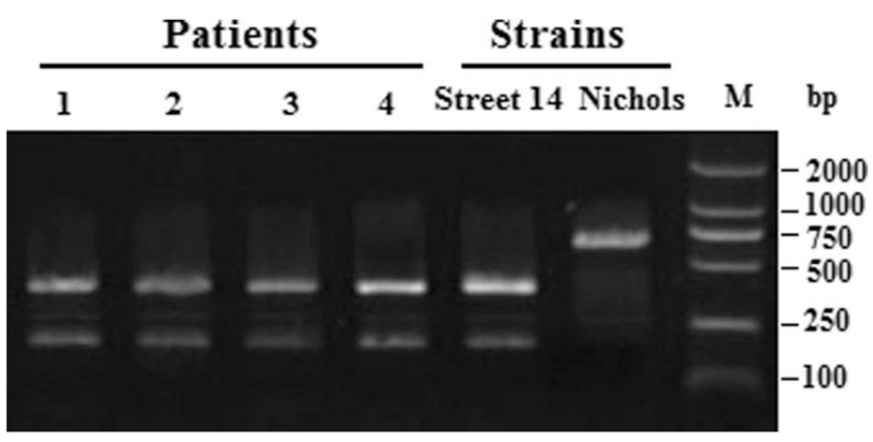

Figure 2.

Restriction enzyme analysis of PCR amplifications showed A2058G mutations in the 23S rRNA gene of T. pallidum from 4 patients' lesions. Street 14 is mutated strain where the PCR product of $23 S$ rRNA gene could be digested into 2 bands, whereas wild-type Nichols strain only shows 1 band. 


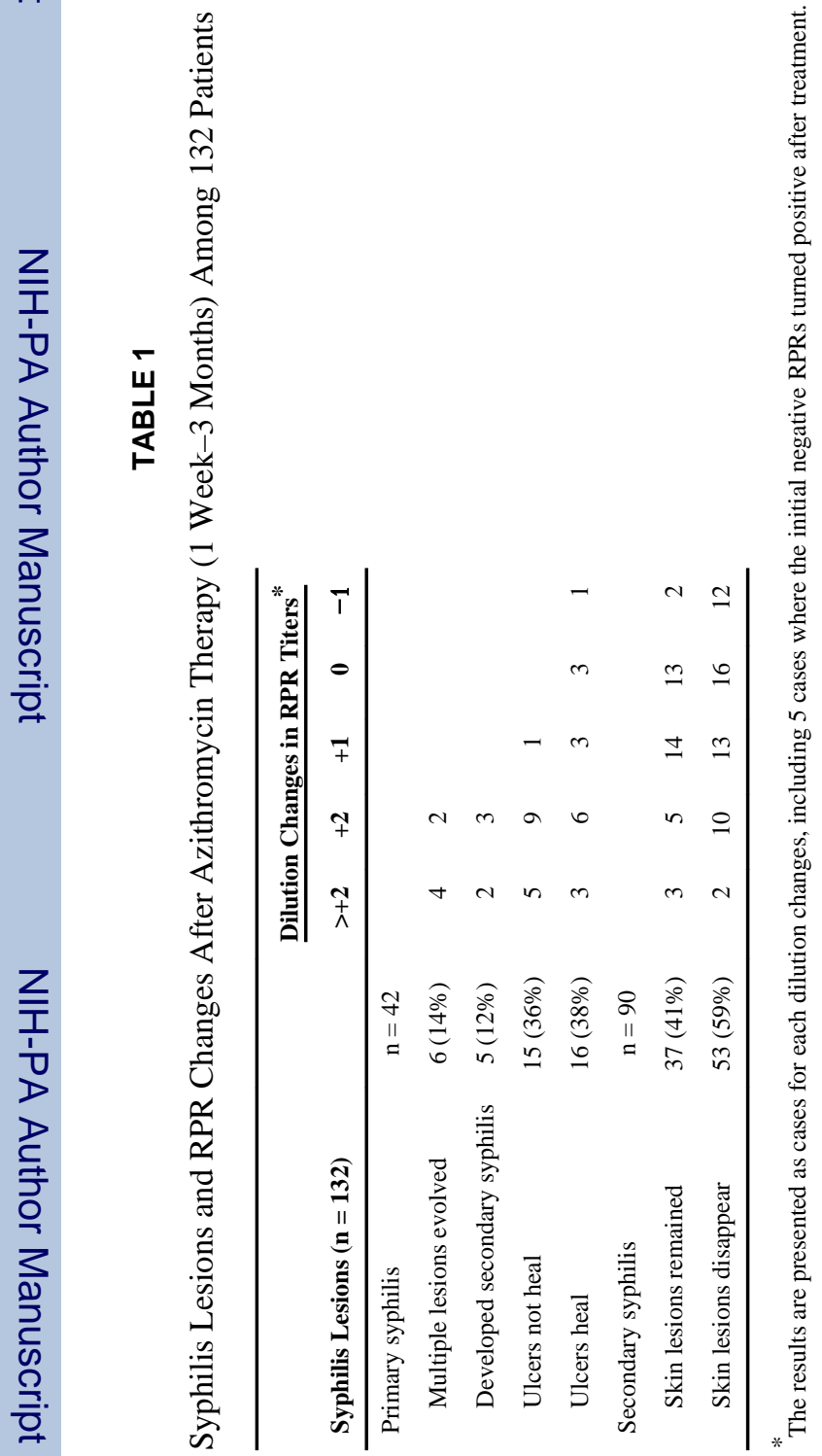




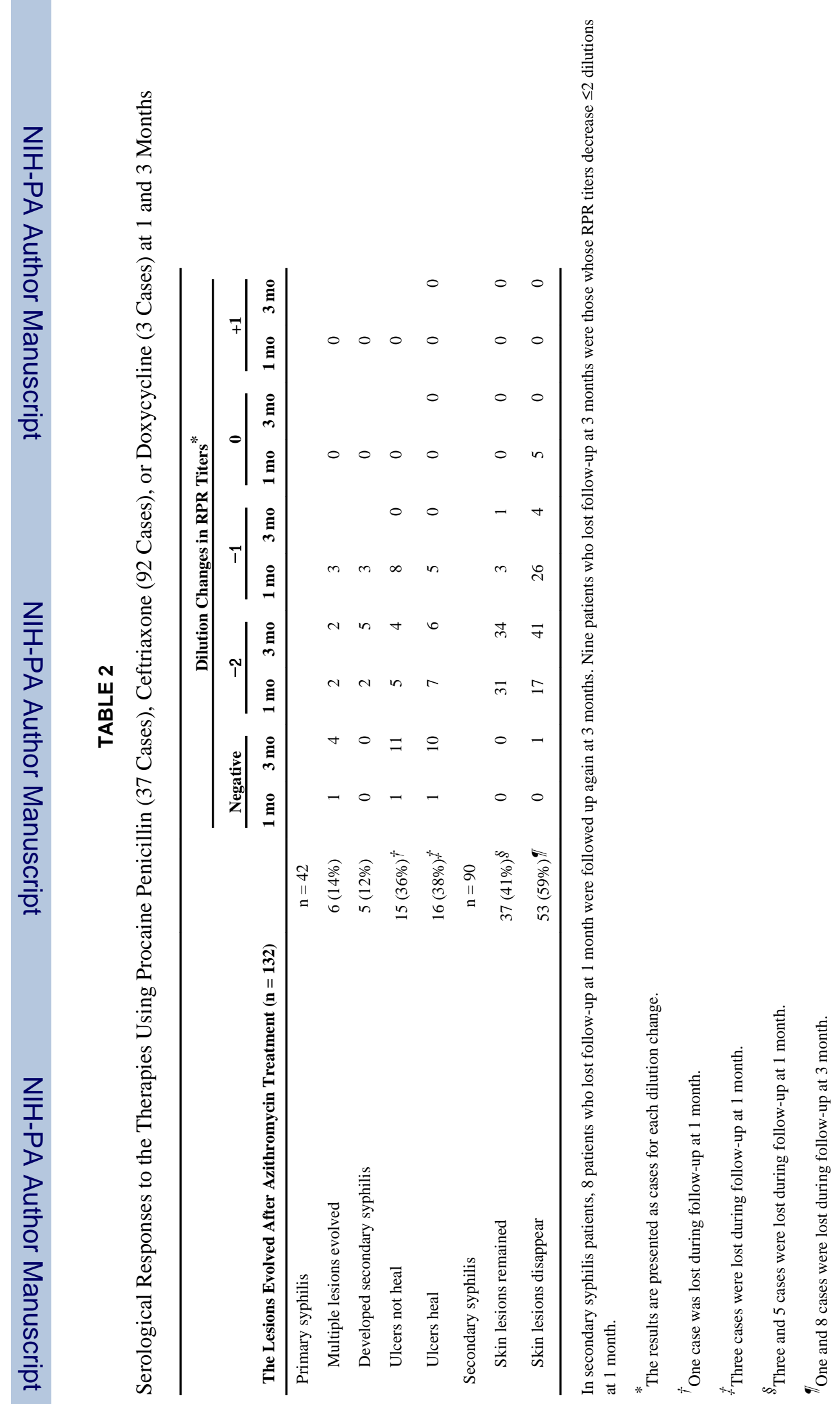

Sex Transm Dis. Author manuscript; available in PMC 2011 November 1. 
TABLE 3

Baseline Characteristics of the Study Population $(n=132)$

\begin{tabular}{|c|c|}
\hline Characteristics & Number $(\%)$ \\
\hline \multicolumn{2}{|l|}{ Sex } \\
\hline Male & $85(64.4)$ \\
\hline Female & $47(35.6)$ \\
\hline \multicolumn{2}{|l|}{ Age } \\
\hline $18-27$ & $38(28.8)$ \\
\hline $28-37$ & $46(34.9)$ \\
\hline $38-47$ & $29(22.0)$ \\
\hline $48-56$ & $9(6.8)$ \\
\hline \multicolumn{2}{|l|}{ Self-reported sexual behaviors } \\
\hline Only homosexual behaviors & $11(8.3)$ \\
\hline Bisexual behaviors & $4(3.0)$ \\
\hline Only heterosexual behaviors & $117(88.6)$ \\
\hline \multicolumn{2}{|c|}{ History of macrolide therapy in the last 6 mo } \\
\hline Yes & $26(19.7)$ \\
\hline No & $64(48.5)$ \\
\hline Not known & $42(31.8)$ \\
\hline \multicolumn{2}{|c|}{ Syphilis stage (before azithromycin treatment) } \\
\hline Primary & $42(31.8)$ \\
\hline Secondary & $90(68.2)$ \\
\hline
\end{tabular}

